\title{
Discordant Twin: Marked Vascular Communication Between Separated Dichorionic Diamniotic Placentas
}

\author{
Saeko Hagihara, Shigeki Matsubara, Tomoyuki Kuwata, Yosuke Baba, Kazuhiko Shimada, \\ Hiroko Yamanaka, Takashi Watanabe, and Mitsuaki Suzuki \\ Department of Obstetrics and Gynecology, Jichi Medical School, Tochigi, Japan
}

W e report on a woman who gave birth to dichorionic diamniotic twins with a birthweight discordancy of $30 \%$. Her placenta exhibited characteristic features. The placental villous tissues were completely separated, but there was marked vascular communication between the two placentas. The lighter twin received less blood than the heavier twin via these vascular communications. Although the details are unknown, this abnormal placental structure may have caused the weight discordancy in this case. Clinicians must pay attention to placental vascular communication as one possible cause of twin discordancy in not only monochorionic but also dichorionic twins.

The association between discordant fetal growth and the complications of twin pregnancy is widely recognized both in monochorionic diamniotic (MCDA) twins and dichorionic diamniotic (DCDA) twins (Cunningham et al., 2001; Minakami et al., 1999; Redman et al., 2002). In the former (MCDA twins), weight discordancy is frequently attributed to twin-twin transfusion syndrome, in which various vascular anastomoses are present, inducing an imbalance in fetal/placental perfusion, leading to a lighter donor/heavier recipient, and finally to weight discordancy (Benirschke \& Kaufmann, 2000; Cunningham et al., 2001). Placental vascular anastomosis, therefore, is considered to induce twin-to-twin transfusion syndrome, a syndrome of twin discordancy unique to MCDA twins.

Twin discordancy occurs not only in MCDA twins but also DCDA twins (Cunningham et al., 2001; Matsubara et al., 2000; Minakami et al., 1999). Many physiological/ pathological conditions induce discordant DCDA twins: Some chromosomal anomalies, congenital infections, a constitutionally smaller twin, and various malformations; When these common disorders occur in only one twin, they may lead to growth restriction of the corresponding twin, and finally to weight discordancy (Benirschke \& Kaufmann, 2000; Cunningham et al., 2001).

Another important cause of discordant DCDA twins is placental abnormality, such as the disproportionately small placental size of one twin (placental size discordancy) and cord abnormalities (velamentous insertion, etc.; Benirschke \& Kaufmann, 2000; Cunningham et al., 2001; Victoria et al., 2001). Large placental vascular anastomoses/communications, if they are present in DCDA twin placentas, may also lead to weight discordancy in DCDA twins, similar to twin-twin transfusion syndrome in MCDA twins. However, communications large enough to induce marked transplacental flow have never been reported in DCDA twin placentas (Benirschke \& Kaufmann, 2000; Robertson $\&$ Neer, 1983). We report here a DCDA twin case, in which large vascular communication was present between macroscopically completely separate DCDA placentas. We consider that this vascular communication may have led to the lighter weight of one twin, and finally to discordancy in this DCDA twin case.

\section{Case Report}

A 37-year-old primigravid woman with twin gestation was admitted to Jichi Medical School Hospital at 24 weeks of gestation because of clinical signs of threatened preterm delivery. She had conceived spontaneously, and an echogram performed during the first trimester revealed two separate placentas with thick dividing membranes and twin-peak signs, characteristic of DCDA placentas. After successful tocolysis treatment with a beta 2-agonist, fetal weight discrepancy of $30 \%$ was diagnosed at 33 weeks of gestation. Repeated echographic examinations indicated that there were two completely separate placentas of approximately the same size and each placental cord was attached at the middle of each placenta. There were no echographically-detectable morphological abnormalities, nor evidence of congenital infections. At 36 weeks of gestation, she gave birth abdominally to female twin infants, weighing $2078 \mathrm{~g}$ and $2980 \mathrm{~g}$, respectively (discordancy rate: 2980-2078 $/ 2980=30.3 \%$ ). Both infants were healthy with no signs of malformation/infection. The mother and babies were discharged at eight days with no complications. Both babies are now doing well with no sequelae.

Placental Findings

Address for correspondence: Shigeki Matsubara, Department of Obstetrics and Gynecology, Jichi Medical School, 3311-1, Minamikawachi-machi, Kawachi-gun, 329-0498 Tochigi, Japan. Email:matsushi@jichi.ac.jp 
There were two separate placentas of approximately the same size, thickness and color (Figure 1; left panel). There were no macroscopically-detectable abnormalities, such as infarctions, hematomas. The right panel of Figure 1 indicates the view from the fetal side. The left placental cord cord (A) - belonged to the lighter twin (A), and the right - cord (B) - to the heavier twin (B). A placental dividing membrane lay across the placenta $(A)$, and did not meet over the area between the two placentas. All the vessels of the upper half of the placenta (A) finally gathered in cord (A), whereas all the vessels of the lower half of the placenta (A) went across the fetal membranes, reaching the other placenta (B). In other words, cord (A) - thus twin (A) received blood only from the upper half of the placenta $(A)$, while cord B - twin (B) - received blood from both the lower half of the placenta (A) and the other placenta (B). No communication was identified between the upper and lower halfs of the placenta (A). Histological examination revealed that the dividing membranes had thick chorion laeve trophoblast layers, confirming the DCDA placenta.

\section{Discussion}

This case showed quite characteristic placental morphology: 1) the placenta was composed of two completely separate villous tissues (separate placentas) with histological examination confirming DCDA placenta, 2) a septal dividing membrane lay across one placenta $(\mathrm{A})$, and 3) all the vessels of the lower half of this placenta (A) directly entered the other placenta $(B)$, while not entering the upper half of the placenta (A). The lighter twin (A), therefore, received blood from only the upper half of one placenta (A), and the heavier twin (B) received blood from both the lower half of one placenta (A) and the whole of the other placenta (B). In brief, the lighter twin (A) received approximately one quarter of the total placental blood supply — half of placenta (A), while the heavier twin (B) received three quarter - half of placenta (A) + the whole of placenta (B). This placental vessel structure may account for the growth restriction of twin (A), and this may have led to the weight discordancy of this DCDA twin. Since we have no direct evidence that there was a direct cause and effect relationship between the placental vascular communication and weight discordancy this remains hypothetical, although reasonable.

The accumulated data indicates that DCDA placentas, even though the two placentas are completely fused and appear macroscopically inseparable, do not have interplacental vessel communications with the very rare exception of tiny vessel communication confined to one cotyledon (Benirschke \& Kaufmann, 2000; Robertson \& Neer, 1983). Benirschke and Kaufmann (2000) described that "despite numerous injection attempts we have never seen anastomoses between the blood vessels of dichorionic twin placentas". Robertson and Neer (1983) reported that, among 68 dichorionic twin placentas, there was no transplacental circulation in 67, and in a single case with transplacental circulation, the anastomoses were limited to one area of a single cotyledon. This is in stark contrast to the observation that MCDA placentas usually possess vascular communications (Benirschke \& Kaufmann, 2000; Robertson \& Neer, 1983). In this case, histological examination confirmed that this twin placenta was a DCDA placenta, and not an MCDA placenta. At present, it is not clear why this DCDA placenta possessed large interplacental vascular communication, but one possible explanation is the presence of an accessory lobe. The smaller twin (A) was supplied by the upper half of placenta (A), and the other twin (B) by placenta (B). Initially, there may have been an accessory lobe of placenta (B), which also supplied blood to twin (B), standing completely separate from the main placenta (B). In the early stage of placental development, therefore, there may have been three definite parts of the

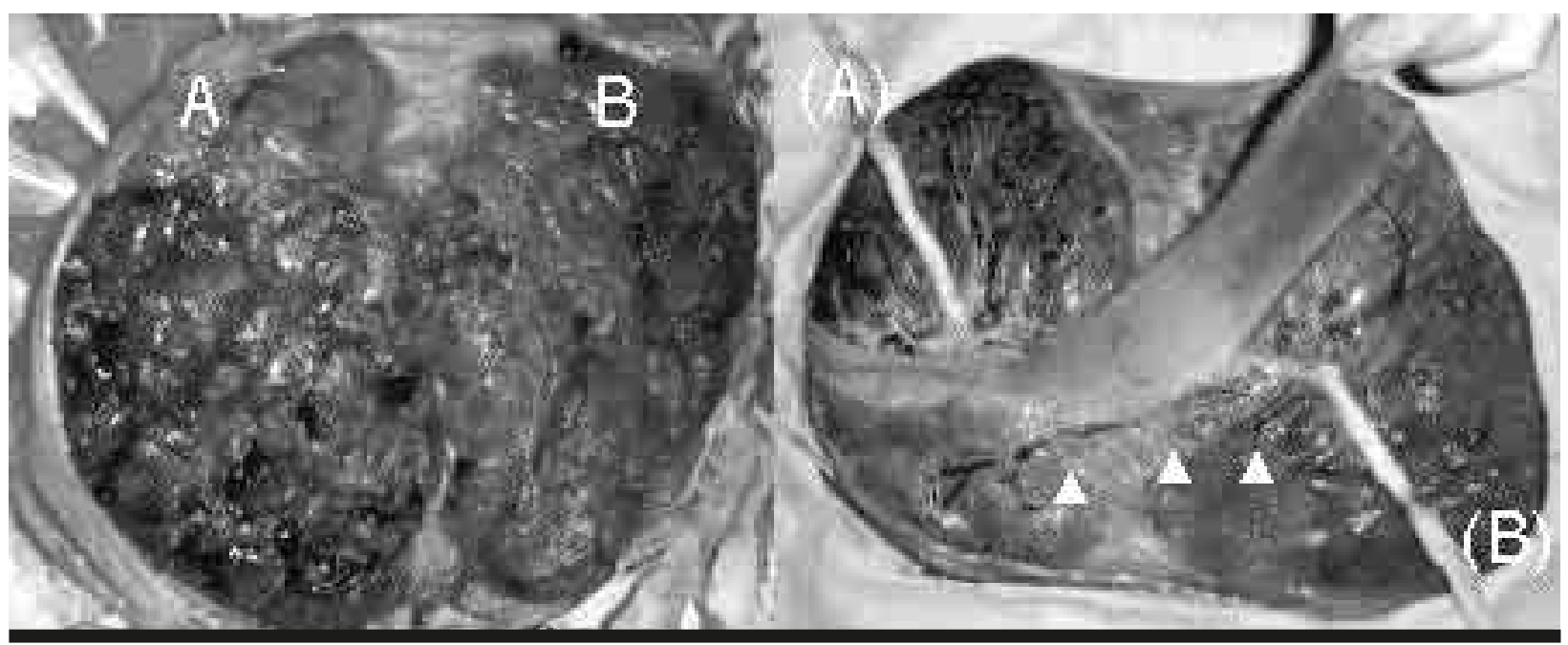

Figure 1

Placental view from the maternal surface (left panel) and the fetal surface (right panel). Whereas the view from the maternal side indicates that the placenta is composed of completely separate placentae $((A)$ and $(B))$, the view from the fetal side indicates marked vascular communication.

(A) in the right panel indicates the placental cord belonging to the lighter twin, and (B) to the heavier twin. The dividing membranes cross the placenta (A). While the placental vessels of the upper half of placenta (A) gathered in cord (A), those of the lower half go across the membranes (arrowheads) and gathered in the other placental cord (B). 
placenta; the placenta for twin (A), an accessory lobe for twin (B), and the main placenta for twin (B). During subsequent placental development, the former two fused to form one inseparable placental structure, which is now recognized as placenta (A). Therefore, the lower half of placenta (A) may be the accessory lobe placenta for twin (B). This model may well explain this observation, although we have no evidence. DNA typing for each part of the placenta may give direct evidence. A vanishing triplet may be another candidate. The lower half of placenta (A) once supplied blood to a third fetus (MCDA twin for twin (B)), which died in utero, leaving the vessel communications. Postpartum examinations, however, revealed no corresponding dead fetus/fetal remnants.

This case is highlighting important clinical suggestions. Weight discordancy in DCDA twins has a close association with poor perinatal/neonatal outcome, especially for the lighter twin (Cunningham et al., 2001; Minakami et al., 1999). Clinicians must identify the causes of discordancy in each case as precisely as possible. In this case, we antenatally determined the placental size and cord insertion site of each twin, considering that placental size discordancy and abnormal cord insertion (i.e., velamentous cord insertion) may cause weight discordancy. However, we found no positive findings; echographically detectable placental size was identical between the two placentas, and the cord was attached to the middle of each placenta, both of which were confirmed by postpartum examination. The absence of apparent placental size discordancy, therefore, cannot preclude the possibility that there are placental problems behind DCDA twin discordancy. Placental vascular communication must be added as one cause of DCDA twin discordancy, even though it rarely occurs and its pathogenesis/pathophysiology awaits further studies.

\section{References}

Benirschke, K., \& Kaufmann, P. (2000) Multiple pregnancy. In K. Benirschke, \& P. Kaufmann (Eds.), Pathology of the human placenta (pp. 790-902). Springer-Verlag: New York.

Cunningham, F. C., Gant, N. F., Leveno, K. J., Gilstrap, L. C., Hauth, J. C., \& Wenstrom, K. D. (2001). Multifetal pregnancy. In F. C. Cunningham, N. F. Gant, K. J. Leveno, L. C. Gilstrap, J. C. Hauth, \& K. D. Wenstrom (Eds.), Williams obstetrics (21 th ed.; pp. 765-810). Conneticut: Appleton \& Lange.

Matsubara, S., Minakami, H., \& Sato, I. (2000). Placenta of discordant twins: Lack of change in histochemically detectable enzyme activities. Twin Research, 3, 123-128.

Minakami, H., Honma, Y., Matsubara, S., Uchida, A., Shiraishi, H., \& Sato, I. (1999). Effects of placental chorionicity on outcome in twin pregnancy: A cohort study. Journal of Reproductive Medicine, 44, 595-600.

Redman, M. E., Blackwell, S. C., Refuerzo, J. S., Kruger, M., Naccasha, N., Hassan, S. S., et al. (2002). The ninety-fifth percentile for growth discordance predicts complications of twin pregnancy. American Journal of Obstetrics and Gynecology, 187, 667-671.

Robertson, E. G., \& Neer, K. J. (1983). Placental injection studies in twin gestation. American Journal of Obstetrics and Gynecology, 147, 170-174.

Victoria, A., Mora, G., \& Arias, F. (2001). Perinatal outcome, placental pathology, and severity of discordance in monochorionic and dichorionic twins. Obstetrics \& Gynecology, 97, 310-315. 